\title{
Modified cantilevers to probe unambiguously out-of-plane piezoresponse
}

\author{
Natalia Alyabyeva ${ }^{1}$, Aimeric Ouvrard ${ }^{1}$, Ionela Lindfors-Vrejoiu ${ }^{2}$, Alexey Kolomiytsev ${ }^{3}$, Maxim \\ Solodovnik ${ }^{3}$, Oleg Ageev ${ }^{4}$ and Damien McGrouther ${ }^{5}$ \\ ${ }^{1}$ Institut des Sciences Moléculaires d'Orsay (ISMO), CNRS, Université Paris-Saclay, F-91405 Orsay, France \\ ${ }^{2} I I$. Physikalisches Institut, Universität zu Köln, 05937 Köln, Germany \\ ${ }^{3}$ Southern Federal University, Department of Nanotechnologies and Microsystems Technology, 347922, 2, \\ Shevchenko Str., Taganrog, Russia \\ ${ }^{4}$ Research and Educational Centre "Nanotechnology", Southern Federal University, 347928, 2, Shevchenko Str., \\ Taganrog, Russia \\ ${ }^{5}$ School of Physics \& Astronomy, University of Glasgow, Glasgow G12 8QQ, UK
}

\begin{abstract}
We demonstrate and investigate the coupling of contributions from both in-plane (IP) polarization and out-of-plane (OP) components in $\mathrm{BiFeO}_{3}$ (BFO) thin films polarization probed by piezoresponse force microscopy (PFM). Such coupling leads to image artefacts which prevent the correct determination of OP polarization vector directions and the corresponding piezoelectric coefficient $\mathrm{d}_{33}$. Using material strength theory with a 1D modelling of the cantilever oscillation amplitude under electrostatic and elastic forces as function of the tip length, we have evidenced the impact of IP piezoresponse to the OP signal for tip length longer than $4 \mu \mathrm{m}$. The IP polarization vector induces a significant longitudinal bending of the cantilever, due to the small spring constant of long tips, which provokes a normal deviation superimposed to the OP piezoresponse. These artefacts can be reduced by increasing the longitudinal spring constant of the cantilever by shortening the tip length. Standard cantilevers with $15 \mu \mathrm{m}$-long tips were modified to reach the desired tip length, using focused ion beam techniques and tested using PFM on the same BFO thin film. Tip length shortening has strongly reduced IP artefacts as expected, while the impact of nonlocal electrostatic forces, becoming predominant for tips shorter than $1 \mu \mathrm{m}$, have led to a nonnegligible deflection offset. For shorter tips, strong electric field from cantilever beam, can induce polarization switching as observed for $0.5 \mu \mathrm{m}$-long tip. Tip length ranging from 1 to $4 \mu \mathrm{m}$ allowed minimizing both artefacts to probe unambiguously OP piezoresponse and quantify the $d_{33}$ piezoelectric coefficient.
\end{abstract}

Keywords: Piezoresponse force microscopy, $\mathrm{BiFeO}_{3}$ thin films, out-of-plane piezoresponse, $\mathrm{d}_{33}$ piezoelectric coefficient, focused ion beam. 


\section{INTRODUCTION}

Piezoresponse force microscopy (PFM) is the most widely employed technique for local characterization of piezoelectric materials. Its principle is based on the converse piezoelectric effect, where an external electric field is applied to the surface of a ferroelectric sample between a sharp conducting tip and a grounded bottom electrode. ${ }^{1-4}$ Induced mechanical expansions or contractions of the sample are detected as shifts of the cantilever oscillation in contact mode of an atomic force microscopy (AFM). Three different signals can be detected: topography, amplitude and phase. The out-of-plane (OP) or the in-plane (IP) piezoresponse of the different domains can be probed using amplitude and phase by monitoring normal or lateral (torsional) cantilever deflections on the four-quadrant photodetector, respectively. Conventionally, amplitude piezoresponse yields a measurable signal, proportional to the piezoelectric coefficients, allowing to probe ferroelectric domain walls and defines the local electromechanical activity of the surface. The phase of electromechanical response of the surface enables the visualization of domains and the determination of polarization vectors directions. ${ }^{1-7}$ If polarization vector is oriented normal to the surface and pointing downward, for a positive (negative) tip bias, it results in a local sample expansion (contraction) and surface oscillations are in phase with tip voltage, phase $=0^{\circ}$, whereas for opposite domains (pointing upward), phase $=180^{\circ} .4$ For polarization vector oriented in the surface plane and pointing leftward, lateral surface oscillations are in phase $\left(0^{\circ}\right)$ with the tip voltage, while for rightward, they are phase shifted $\left(180^{\circ}\right)$. It is widely known, that PFM images can often be influenced by sources of additional cantilever forces that give rise to artefacts. ${ }^{4-7}$ For example, the impact of non-local (from the cantilever beam) and local (under the tip) electrostatic forces can strongly perturb PFM signals by changing the dynamics of "cantilever-sample" interaction. ${ }^{4-10}$ In this case, electrostatic and elastic interactions between the charged cantilever and sample dipoles induce additional contributions to PFM signals leading to weakened phase contrast (less than $180^{\circ}$ phase change across oppositely oriented domains)., ${ }^{\mathbf{9 1 0}}$ Dealing with such artefacts requires modified experimental strategies to reduce levels of additional forces, through: (i) a careful selection of imaging parameters, possibly employing the use of DC voltage or/and changing regulation (driving) frequency, 9,11 (ii) the calibration of the laser beam position at the highest cantilever elevation giving the best piezoresponse sensitivity, ${ }^{12}$ or (iii) the use of cantilevers with high normal spring constant for OP PFM, by changing beam geometry (length

and thickness). ${ }^{\mathbf{1 , 2 , 6}}$ In this work, we highlight the influence of IP polarization contributions through elastic forces on OP PFM signals and demonstrate a method to reduce them. We have modified 
standard PFM cantilevers using a focused ion beam instrument, to create shorter tips that reduce artefacts and enhance OP piezoresponse sensitivity when imaging ferroelectric domain structure in a $\mathrm{BiFeO}_{3}$ thin film. We show, with the help of material strength theory and cantilever dynamic calculations under electrostatic and elastic perturbations, that the tip length plays an important role.

\section{EXPERIMENTAL SETUP}

In order to study how to reduce the influence of electrostatic and elastic perturbations in PFM measurements, we have used a $\mathrm{BiFeO}_{3}$ (BFO) thin film as a test sample. $60 \mathrm{~nm}$-thick $\mathrm{BFO}$ thin epitaxial film was grown on $\mathrm{SrRuO}_{3}$ buffer layer on $\mathrm{DyScO}_{3}(110)$ substrate by pulsed laser deposition in a $0.14 \mathrm{mbar}_{2}$ atmosphere with a substrate temperature of $650^{\circ} \mathrm{C}$. Laser pulse energy of $90 \mathrm{~mJ}$ (energy density of $0.4 \mathrm{~J} / \mathrm{cm}^{2}$ ) was used, leading to a $0.5-1.5 \mathrm{~nm} / \mathrm{min}$ growth rate. ${ }^{13}$ Further details of the BFO thin film deposition and its crystallinity investigation by XRD are given elsewhere. ${ }^{14}$ All OP and IP PFM measurements were performed using a scanning probe microscope in air condition (Solver Next, NT-MDT). BFO thin film was investigated along [010], [100] and [1̄10] crystallographic orientations using standard commercial cantilevers (MFM01 $\mathrm{CoCr}, 15 \mu \mathrm{m}$ tip length, NT-MDT) with normal spring constant $\mathrm{k}_{\mathrm{Norm}}=5 \mathrm{~N} / \mathrm{m}$ and subsequently modified cantilevers to evidence electrostatic and elastic artefacts. $\mathrm{V}_{\mathrm{AC}}=1 \mathrm{~V}$ was applied to the cantilever to create a polarizing electric field $\left(\mathrm{V}_{\mathrm{DC}}=0 \mathrm{~V}\right)$. The modulation frequency was set at $\mathrm{f}=100 \mathrm{kHz}$, off contact resonance $(85 \mathrm{kHz})$ to reduce topography contribution to PFM signals while keeping a good sensitivity for amplitude and phase signals. ${ }^{11,15}$ PFM amplitude in NT-MDT microscopes is in "nA". We have used force-distance characteristics in contact mode on our BFO thin film to convert the deflection in " $\mathrm{AA}$ " to " $\mathrm{nm}$ ". ${ }^{16}$ In our system, phase is renormalized, then for polarization vector pointing downward or leftward (upward or rightward), the phase equals to $-90^{\circ}\left(90^{\circ}\right)$. To be able to investigate the same area, several marker points were done on the sample surface allowing to recover the initial position by optical microscope. The exact area was found by scanning first at large scale $\left(20 \times 20 \mu \mathrm{m}^{2}\right)$ before zooming.

The optimal tip length for OP PFM measurements was determined based on material strength theory ${ }^{17,18}$ and a 1D beam model of the cantilever dynamic, ${ }^{6,19}$ by taking into account non-local and local electrostatic forces, elastic forces and by introducing OP and IP piezoresponses. Based on the performed calculations, new cantilevers with two different tip lengths $(1.5$ and $0.5 \mu \mathrm{m})$ were prepared using focused ion beam techniques (FIB) (Nova NanoLab 600, FEI). Preparation consisted of several steps of ion-beam etching and FIB-induced carbon chemical vapour deposition 
(C-CVD). ${ }^{20,21}$ As a first step, the tip of a standard cantilever was removed using FIB etching with $30 \mathrm{keV}, 1.5 \mathrm{nA}$ ion beam parameters and a $-10^{\circ}$ tilt angle relative to the cantilever surface normal. At the second step, a conical structure was grown using C-CVD. The conical shape of the tip base allows increasing both tip stability and life time. C-CVD was performed at a $52^{\circ}$ tilt angle relative to the cantilever normal. For the $1.5 \mu \mathrm{m}$-long tip, six sequential concentric disks were deposited by varying the ion beam current to achieve progressively smaller diameters (30 pA: (1) $2.5 \mu \mathrm{m}$, (2) $2 \mu \mathrm{m}$, (3) $1.5 \mu \mathrm{m}, 10 \mathrm{pA}$ : (4) $1 \mu \mathrm{m}, 1 \mathrm{pA}$ : (5) $0.5 \mu \mathrm{m}$ and (6) $0.2 \mu \mathrm{m}$ ), creating a conical base. At the final step, a sharp tip extremity was formed by FIB etching with 1 pA beam current. The final conical structure had a $1.5 \mu \mathrm{m}$ length. For the shorter tip, only disk (1), (4), (5) and (6) were grown and etched at the same ion beam parameters of the previous tip for a total $0.5 \mu \mathrm{m}$ tip length. FIB prepared cantilevers were tested using spreading resistance AFM measurements on silicon samples having a known resistivity and compared to commercial cantilevers. It was found that FIB cantilevers provides good conductivity comparable with $\mathrm{CoCr}$ coated standard commercial cantilevers. Modified cantilevers have 10 to 100 utilisation cycles, depending on AFM regulation parameters. ${ }^{21}$ Scanning electron microscopy (SEM) was used to image FIB prepared tips.

\section{EXPERIMENTAL RESULTS}

\section{A. Observation of artefacts in OP piezoresponse of BFO thin film}

Figure 1 presents OP and IP PFM measurements of BFO thin film piezoresponse obtained with a standard (15 $\mu \mathrm{m}$-long tip) cantilever. ${ }^{22}$ Parallel stripe domains $\left(D_{1}\right.$ and $\left.D_{2}\right)$ with $200 \mathrm{~nm}$ width are observed along the $[0 \overline{1} 0]$ direction.

OP piezoresponse: Figure 1(a-c) shows the induced normal sample piezoresponse, giving a direct access to the OP distribution of the ferroelectric domains (FE) along the [010], [100] and [1̄10] directions. According to the OP PFM operation principle, bright regions should correspond to positively charged domains, i.e upward polarization vector (positive phase shift), while dark ones should correspond to negatively charged domains, i.e downward polarization. In our observation, different piezoresponse contrasts were obtained for different azimuthal sample orientation, which is in contradiction with OP PFM principle. ${ }^{\mathbf{1 , 1 1}}$ 


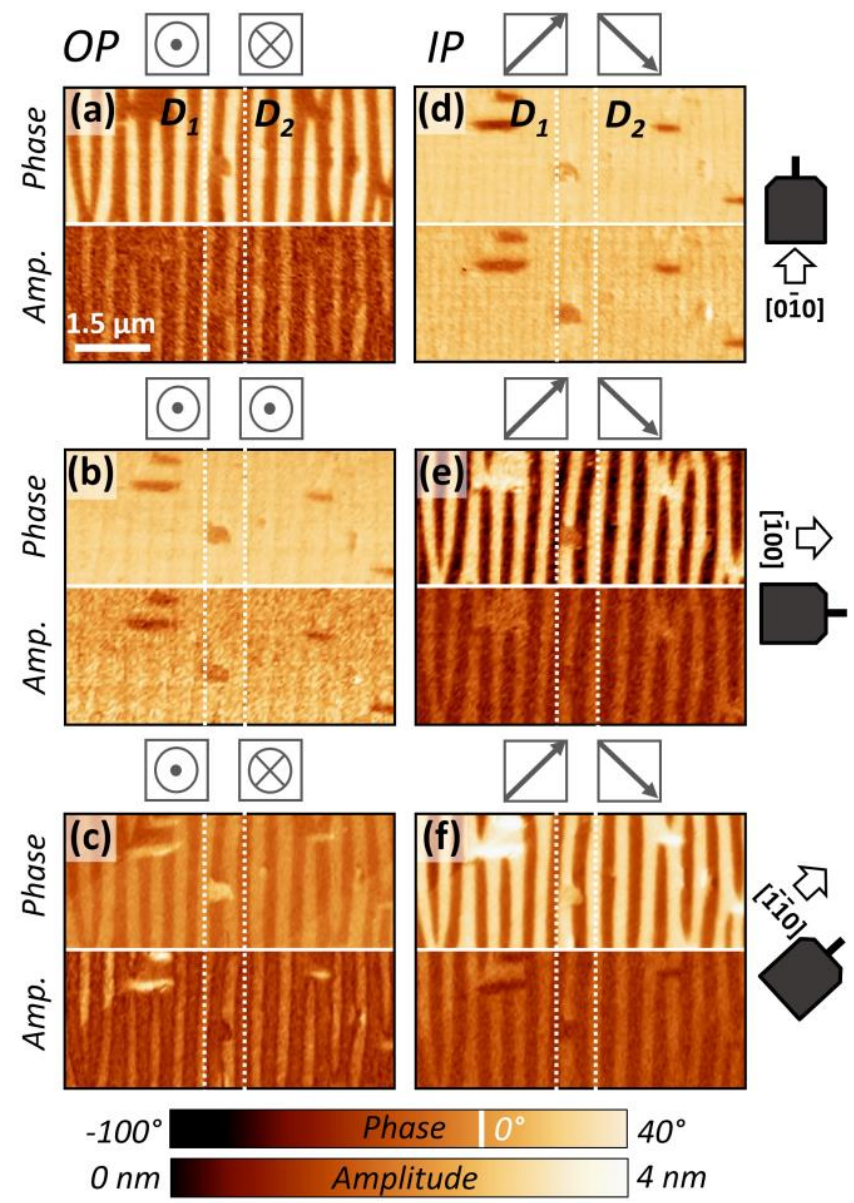

FIG. 1. PFM images of FE domains in BFO thin film piezoresponse obtained with a standard $(15 \mu \mathrm{m}$-long tip) cantilever: (a-c) OP and (d-f) IP components of the piezoresponse. PFM scanning direction along (a, d) [0 $\overline{1} 0]$,

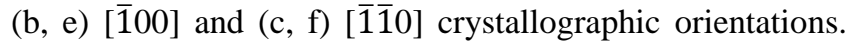
Domains $D_{1}$ and $D_{2}$ are highlighted by white dotted lines. Cantilever position relative to domains and crystallographic directions are given.

Our results, in Fig. 1(a) and Fig. 1(c), strongly suggest the presence of artefacts that modify the expected domain contrast. Only Fig. 1(b) resembles the expected domain contrast ${ }^{13,14}$ but with a significantly weaker phase response and domain walls almost absent in the amplitude signal.

IP piezoresponse: In Fig. 1(d-f) are shown the induced lateral piezoresponse measured in the

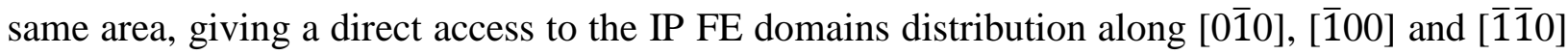
directions, respectively. The bright regions $\left[D_{1}\right.$ and $D_{2}$ in Fig. $1(\mathrm{~d}) ; D_{2}$ in Fig. 1(e, f)] correspond to rightward IP piezoresponse direction (positive phase shift). The dark region $\left[D_{l}\right.$ in Fig. 1(e)] corresponds to the leftward IP piezoresponse direction (negative phase shift). The $D_{l}$ phase shift in Fig. 1(f) is close to $0^{\circ}$ because the polarization vector is collinear to the scan direction. 
Through consideration of Fig.1(b) and Fig. 1(d-f), polarization vector directions were deduced, allowing the determination of the orientation of both $D_{1}$ and $D_{2}$ domains, $71^{\circ}$ relative to one another, [inserts in Fig. 2(a)] in agreement with literature. ${ }^{13,14,22}$ The magnitude of the phase shift is less than $180^{\circ}$ and the presence of domain pattern contrast for both amplitude and phase in OP PFM images [Fig. 1(a, c)] similar to the IP contrast [Fig. 1(e, f)] is consistent with mixed sensitivity to both OP and IP polarization components. Additionally, the walls between domains cannot be clearly visualised. It must be noticed that similar measurements have been repeated for different driving frequencies (30 to $150 \mathrm{kHz}$ ) of the cantilever (not presented here) and reveal the presence of artefacts at any frequency on the OP piezoresponse. The frequency only affected the PFM sensitivity for amplitude and phase.

\section{B. Origin of artefacts in the OP piezoresponse}

In order to understand the origin of the sensitivity to both OP and IP components, we have simulated the normal cantilever oscillation amplitude and the effect of tip length on OP PFM sensitivity in BFO thin film. Both non-local/local electrostatic and elastic forces, and OP/IP contributions were taken into account. A schematic representation of the cantilever showing the different contributing forces is given in Fig. 2(a). First, we have determined normal $\left(\mathrm{k}_{\text {Norm }}\right)$ and longitudinal $\left(\mathrm{k}_{\text {Long }}\right)$ spring constants of cantilevers with tip length ranging from 15 to $0.5 \mu \mathrm{m}$, using material strength theory in analogy with the method of Neumeister and Ducker ${ }^{17}$ [Fig. 2(b)]. Geometrical cantilever parameters are given in Fig. 2(a) and Table 1.

TABLE 1 - Geometrical parameters of standard cantilevers

\begin{tabular}{ccccccc}
\hline \hline $\begin{array}{c}\text { Tip } \\
\text { length } \\
\mathrm{h}(\mu \mathrm{m})\end{array}$ & $\begin{array}{c}\text { Beam } \\
\text { length } \\
\mathrm{L}(\mu \mathrm{m})\end{array}$ & $\begin{array}{c}\text { Beam } \\
\text { width } \\
\mathrm{w}(\mu \mathrm{m})\end{array}$ & $\begin{array}{c}\text { Distance from tip } \\
\text { to beam extremity } \\
\mathrm{d}(\mu \mathrm{m})\end{array}$ & $\begin{array}{c}\text { Beam } \\
\text { thickness } \\
\mathrm{t}(\mu \mathrm{m})\end{array}$ & $\begin{array}{c}\text { Young's } \\
\text { modulus } \\
\mathrm{E}(\mathrm{GPa})\end{array}$ & $\begin{array}{c}\text { Poisson } \\
\text { coefficient } \\
v\end{array}$ \\
\hline 15 & 130 & 35 & 5 & 2 & 131 & 0.266 \\
\hline \hline
\end{tabular}

Figure 2(b) shows that the tip length has a strong impact on the cantilever longitudinal spring constant, while the normal spring constant is completely insensitive. ${ }^{17,18}$ Using this model, we have found that $\mathrm{k}_{\mathrm{Norm}}=4.69 \mathrm{~N} / \mathrm{m}$ for commercial cantilever in agreement with provider specifications. ${ }^{16}$ Decreasing the tip length (h) from 15 to $0.5 \mu \mathrm{m}$ leads to a strong increase in the longitudinal spring constant from $\sim 10^{2}$ to $\sim 10^{5} \mathrm{~N} / \mathrm{m}$, respectively. 

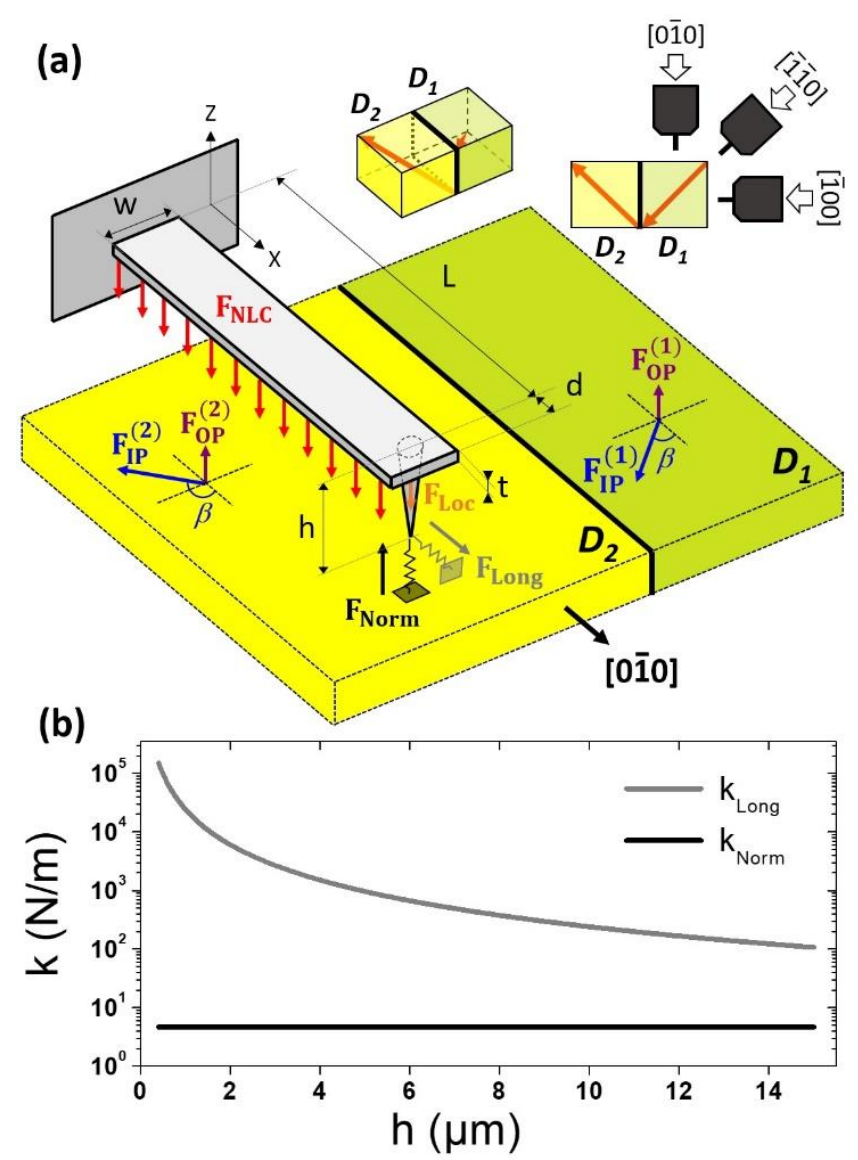

FIG. 2. (a) Schematic representation of the cantilever-sample system in PFM mode with in insert the 3D and 2D representation of the unit cell polarization vectors for $D_{l}$ and $D_{2}$ domains relative to the cantilever orientation. (b) Normal $\left(\mathrm{k}_{\text {Norm }}\right)$ and longitudinal ( $\mathrm{k}_{\text {Long }}$ ) spring constants of the cantilever as a function of tip length.

Secondly, the obtained spring constants were used to simulate the cantilever oscillation amplitude under electrostatic and elastic forces in PFM mode, based on reported methods by Song and Bhushan ${ }^{19}$ and Jesse et al. ${ }^{6}$ and taking into account the direction of IP polarization vectors. The angle of the cantilever beam to the sample surface was set to $0^{\circ}$, in order to simplify the model and highlight PFM imaging mechanisms. The interaction between the cantilever and the sample can be defined by normal and longitudinal springs as represented in the 1D beam model in Fig. 2(a), ${ }^{23,6,17}$ with the vertical bending of cantilever $(\phi)$ described by the following equation: ${ }^{6}$

$$
\frac{\mathrm{d}^{4} \phi(\mathrm{x})}{\mathrm{dx}^{4}}-\kappa^{4} \phi(\mathrm{x})=\frac{\mathrm{q}_{0}}{\mathrm{EI}}
$$

where $\phi(\mathrm{x})$ is the vertical bending of the beam at position $\mathrm{x}$; E is the cantilever Young's modulus, $\mathrm{I}=\mathrm{wt}^{3} / 12$ is the moment of inertia; ${ }^{17,18} \kappa$ is the cantilever eigenvalue for vertical bending 
$\left(\kappa^{4}=\mathrm{f}^{2} \rho \mathrm{S}_{\mathrm{c}} /(\mathrm{EI})\right)^{6,19}$, where $\mathrm{S}_{\mathrm{c}}=\mathrm{wt}$ is the cantilever beam cross section area and $\rho=2.65 \mathrm{~g} / \mathrm{cm}^{3}$ is the cantilever mass density. ${ }^{24} \mathrm{q}_{0}$ is the first harmonic of the distributed load, that can be seen as a non-local capacitive force $\left(\mathrm{F}_{\mathrm{NLC}}\right)$ generated between the beam and the sample surface that is tip length dependent:

$$
\mathrm{q}_{0}=\frac{\varepsilon_{0} \mathrm{SV}_{\mathrm{AC}}\left(\mathrm{V}_{\mathrm{DC}}-\mathrm{V}_{\text {surf }}\right)}{2(\mathrm{~L}+\mathrm{d}) \mathrm{h})^{2}}
$$

where $\varepsilon_{0}$ is the electric permittivity; $S=(L+d) w$ is the cantilever surface; $V_{D C}=0 \mathrm{~V}$ is the offset voltage; $\mathrm{V}_{\text {surf }}=0.5 \mathrm{~V}$ is the surface potential measured by Kelvin force microscopy with standard cantilever. The solution of equation (1) is the following:

$$
\phi(x)=C_{1} e^{\kappa x}+C_{2} e^{-\kappa x}+C_{3} \cos (\kappa x)+C_{4} \sin (\kappa x)+\frac{q_{0}}{f^{2} \rho S}
$$

$\mathrm{C}_{1}, \mathrm{C}_{2}, \mathrm{C}_{3}, \mathrm{C}_{4}$ are constants determined by boundary conditions: ${ }^{\mathbf{6 , 1 9}}$

$$
\left.\phi\right|_{\mathrm{x}=0}=0,\left.\quad \phi^{\prime}\right|_{\mathrm{x}=0}=0,\left.\quad \operatorname{EI}^{\prime \prime}\right|_{\mathrm{x}=\mathrm{L}}=\mathrm{F}_{\mathrm{x}} \mathrm{h},\left.\quad \operatorname{EI}^{\prime \prime \prime}\right|_{\mathrm{x}=\mathrm{L}}=-\mathrm{F}_{\mathrm{z}}
$$

$\mathrm{F}_{\mathrm{X}}$ and $\mathrm{F}_{\mathrm{z}}$ are the projections of the elastic $\left(\mathrm{F}_{\mathrm{Norm}}\right.$ and $\left.\mathrm{F}_{\text {Long }}\right)$ and local electrostatic $\left(\mathrm{F}_{\text {Loc }}\right)$ forces and contributions of $\mathrm{OP}\left(\mathrm{F}_{\mathrm{OP}}\right)$ and $\mathrm{IP}\left(\mathrm{F}_{\mathrm{IP}}\right)$ piezoelectric forces along the $\mathrm{X}$ and $\mathrm{Z}$ axis:

$$
\mathrm{F}_{\mathrm{x}}=\mathrm{F}_{\text {Long }}+\mathrm{F}_{\mathrm{IP}} \cos (\beta) \text { and } \mathrm{F}_{\mathrm{z}}=\mathrm{F}_{\mathrm{Norm}}+\mathrm{F}_{\text {Loc }}+\left|\mathrm{F}_{\mathrm{OP}}\right|
$$

$\beta$ is the angle between the IP polarization vector of $D_{1}$ and $D_{2}$ in BFO and the beam axis. $\beta$ is depending on the crystallographic orientation of the sample relative to the cantilever according to Fig. 2(a): [010] $\beta_{D 1}=\pi / 4, \beta_{D 2}=3 \pi / 4 ;[\overline{1} 00] \beta_{D 1}=-\pi / 4, \beta_{D 2}=\pi / 4$ and [1]10] $\beta_{D 1}=$ $0, \beta_{D 2}=\pi / 2 ; F_{N o r m}$ and $F_{\text {Long }}$ are the normal and longitudinal elastic spring forces, defined by their $\Delta_{\text {Norm }}$ and $\Delta_{\text {Long }}$, the normal and longitudinal cantilever deviations, respectively: ${ }^{19}$

$$
\mathrm{F}_{\text {Norm }}=-\mathrm{k}_{\text {Norm }} \Delta_{\text {Norm }} \quad \text { and } \quad \mathrm{F}_{\text {Long }}=-\mathrm{k}_{\text {Long }} \Delta_{\text {Long }} \text {, }
$$

$\Delta_{\text {Long }}$ and $\Delta_{\text {Norm }}$ are related to $\phi(\mathrm{x})$ by: ${ }^{19}$

$$
\Delta_{\text {Long }}=\left.\mathrm{h} \phi^{\prime}\right|_{\mathrm{x}=\mathrm{L}} \text { and } \Delta_{\mathrm{Norm}}=\left.\phi\right|_{\mathrm{x}=\mathrm{L}}
$$

The local electrostatic force $\left(\mathrm{F}_{\text {Loc }}\right)$ generated between the tip extremity and the sample surface [Fig. 2(a)] is not tip length dependent. Based on the work of Kalinin and Bonnell, ${ }^{\mathbf{5}}$ the magnitude of $\mathrm{F}_{\mathrm{Loc}}$ is estimated to be around $31 \mathrm{nN}$, assuming a tip radius of $35 \mathrm{~nm}$, a tip-sample distance of $0.1 \mathrm{~nm}$ and a local surface potential around $300 \mathrm{mV}$ for a BFO relative dielectric permittivity of 100. The tip-sample distance may vary from 0.1 to $1 \mathrm{~nm}$ in PFM measurements. ${ }^{5}$ We have chosen the smallest value to make $\mathrm{F}_{\mathrm{Loc}}$ not negligible. Note, that the contribution of the Coulombic tipsurface interaction due to polarization charge was excluded as it is completely screened in air. ${ }^{8}$ 
OP piezoelectric force was determined as: $\left|F_{O P}\right|=\left|-k_{N o r m} d_{33} V_{A C} Q\right|=47 n N$ for $d_{33}$ the piezoelectric coefficient set to $100 \mathrm{pm} / \mathrm{V}$ in agreement with literature $\mathbf{2 4 , 2 5}^{2}$ and $\mathrm{Q}=100$ is a preamplifier gain (so-called "quality factor") of the cantilever response allowing to improve the piezoresponse signal to noise ratio (option accessible in the microscope software). The modulus of $\mathrm{F}_{\mathrm{OP}}$ has been introduced in the model in order to reproduce the experimental behaviour, where OP PFM amplitude is not sensitive to the OP vector direction. IP piezoelectric force contributing to the cantilever deflection amplitude was estimated as $F_{I P}=250 \mathrm{nN}$. This value was chosen to fit with in-plane induced artefacts observed for $15 \mu \mathrm{m}$-long tip during OP PFM measurements [Fig. 1(a, c)].

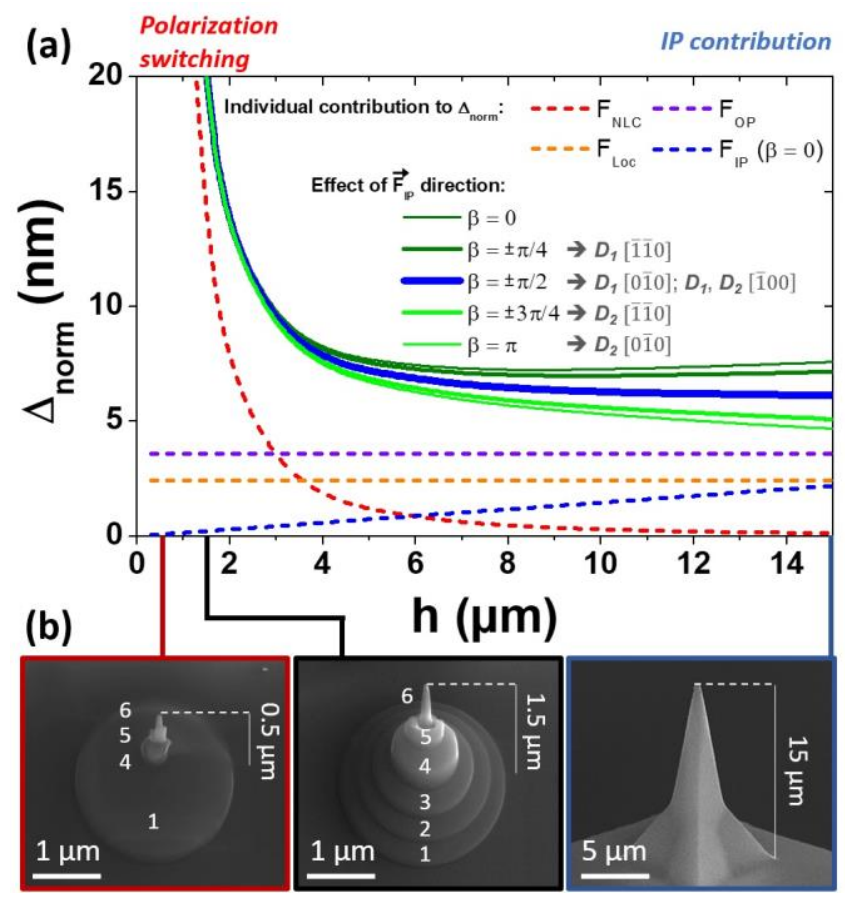

FIG. 3. (a) Normal cantilever amplitude deviation under the impact of electrostatic and elastic forces in addition with the contribution of OP and IP piezoresponses as a function of the tip length. Dashed lines correspond to individual contributions while continuous lines stand for all contributing forces but for different $\beta$. (b) SEM images of the modified cantilevers $(0.5,1.5 \mu \mathrm{m}$ tip length $)$ and the standard $15 \mu \mathrm{m}$ tip length. The preparation parameters corresponding to the disk labels are given in the experimental setup section.

Figure 3(a) shows the calculated vertical deflection amplitude of the cantilever $\left(\Delta_{\text {Norm }}\right)$ as a function of the tip length from 0.5 to $15 \mu \mathrm{m}$. The dashed lines plot the individual contributions from different forces. Contribution of $\mathrm{F}_{\mathrm{OP}}$ and $\mathrm{F}_{\mathrm{Loc}}$ are obviously not tip length dependent and their contributions to $\Delta_{\mathrm{Norm}}$ are around 3.6 and $2.3 \mathrm{~nm}$, respectively. On contrary, $\mathrm{F}_{\mathrm{NLC}}$ becomes 
the predominant force for tip length shorter than $3 \mu \mathrm{m}$, while the effect of $F_{I P}$ (in the case of $F_{I P}$ collinear to the cantilever beam) is in the same order of magnitude of $F_{O P}$ and $F_{\text {Loc }}$ for long tip and continuously decreases for smaller tip lengths. A set of five calculations for different $\beta$, taking into account all contributions were performed to reproduce the PFM observations in Fig. 1(a-c) [continuous lines in Fig. 3(a)]. We have observed that the IP piezoresponse contributions in OP signal are non-negligible for tips longer than $4 \mu \mathrm{m}$, while for shorter tips, it has a minor effect.

\section{Reduction of artefacts in the OP piezoresponse by using shorter tip length}

Figure 4 presents the OP piezoresponse (phase and amplitude along [0 $\overline{1} 0]$ and amplitude along [100]) of $D_{1}$ and $D_{2}$ domains of the same BFO thin film measured by standard $15 \mu \mathrm{m}$ and modified $1.5 \mu \mathrm{m}$-long tips. While for the long tip, a phase contrast was observed between domains, it has totally disappeared for the modified tip as expected for OP phase shift, since $D_{1}$ and $D_{2}$ were both upward polarized [Fig. 4(a)]. As observed in Fig. 4(b), by reducing the tip length, the artificial domain contrast in OP amplitude was reduced and domain walls became visible. In addition, in Fig. 4(c), the use of a short tip allowed a better differentiation between domains and domain walls. A $4 \pm 1 \mathrm{~nm}$ deflection contrast was measured. It can be noticed that the use of $1.5 \mu \mathrm{m}$-long tip increases the offset deflection from 3 to $15 \mathrm{~nm}$, due to the increase of non-local electrostatic forces.

Cantilevers with different tip lengths $(15,1.5$ and $0.5 \mu \mathrm{m})$ have been used to probe domains having a different OP orientation (upward/downward). All measurements were performed in the same region with a modified area, created by scanning in contact-mode under an applied DC voltage of $-4.5 \mathrm{~V}$ to switch $\mathrm{OP}$ polarization from up to downward. Figure 5 shows the phase of the OP piezoresponse. All scans were done along [010] direction with $\mathrm{V}_{\mathrm{DC}}=0 \mathrm{~V}$. As previously demonstrated in Fig. 1(a) and Fig. 4(a, b), for the standard $15 \mu \mathrm{m}$-long tip, the OP piezoresponse shows a contribution of IP domain contrast in both switched/un-switched areas [highlighted by yellow dotted lines in Fig. 5(a)]. 

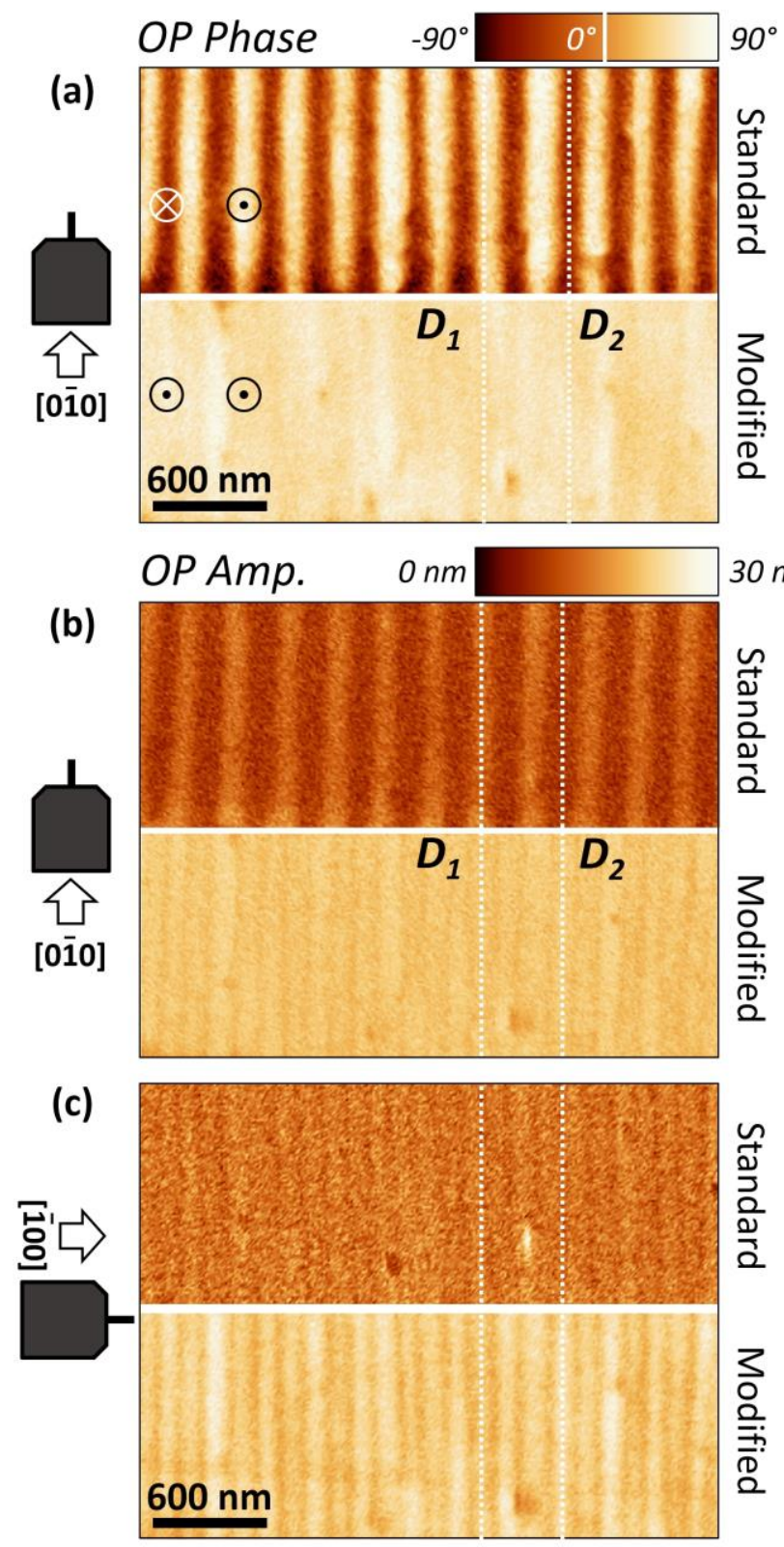

FIG. 4. OP PFM images of $D_{1}$ and $D_{2}$ domains in BFO thin film obtained by standard and modified cantilevers: (a) phase shift for [0 $\overline{1} 0]$ sample orientation, (b) amplitude signal for

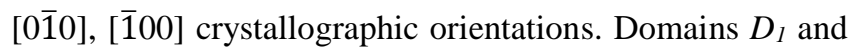
$D_{2}$ are highlighted by white dotted lines. Cantilever position relative to domains and crystallographic directions are given. 

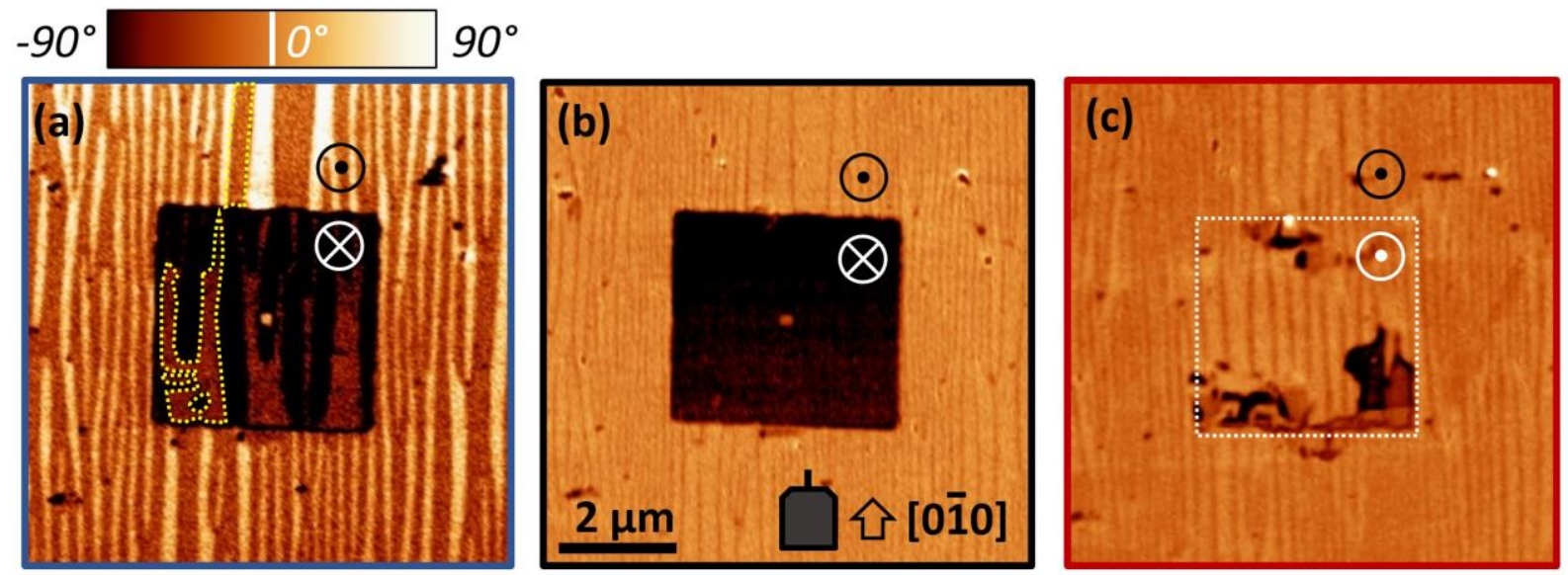

FIG. 5. Impact of the tip length on OP PFM phase imaging of FE domains in BFO thin film: (a) standard cantilever with a $15 \mu \mathrm{m}$-long tip. Dotted yellow contour highlights IP artefacts. (b, c) Modified cantilevers with 1.5 and $0.5 \mu \mathrm{m}$-long tip, respectively. The dotted white frame highlights the area with tip-induced switched polarization.

For the $1.5 \mu \mathrm{m}$-long tip, in Fig. 5(b), the IP contributions have disappeared and phase domain contrast range is close to $180^{\circ}$. Using the shortest tip, polarization switching in the modified area back to upward direction was observed [highlighted by white dotted frame in Fig. 5(c)]. The origin of this effect is not yet clearly understood. The electric field in the tip-surface junction is around $10^{8} \mathrm{~V} / \mathrm{m}$ for any tip used in this work, low enough to avoid polarization switchin. ${ }^{26}$ On the other hand, reducing the tip length increases the electric field in between the beam and the surface of more than $10^{7} \mathrm{~V} / \mathrm{m}$. The addition of both local and non-local electric fields may lead to a polarization switching in the modified area for the shortest tip.

\section{DISCUSSION}

OP PFM results obtained with a standard conductive cantilever of $15 \mu \mathrm{m}$ tip length were neither in good agreement with previously reported results on BFO thin film where both domains are upward polarized, ${ }^{13,14,21}$ nor with the PFM principle where OP piezoresponse should not be crystallographic-direction dependent. ${ }^{11}$ This has motivated the modelling of the normal cantilever oscillation amplitude that shows that the tip length is a critical parameter in OP PFM measurements in agreement with our experimental observations. For $15 \mu \mathrm{m}$-long tip, the calculated contrast for domains having IP orientation $\beta_{D 1}=\pi / 4$ and $\beta_{D 2}=3 \pi / 4$ is $2.1 \mathrm{~nm}$ in a perfect agreement with the amplitude contrast presented in Fig 1(a) and 4(b). The model is able to reproduce all contrasts observed along the different crystallographic directions [Fig. 3(a)]. Indeed, for $\beta_{D 1}=-\pi / 4$ and $\beta_{D 2}=\pi / 4$, no contrast difference in between domains is found, in agreement with PFM results in 
Fig. 1(b) and 4(c). The calculated and experimental contrasts are very similar, around $1.4 \mathrm{~nm}$, for $\beta_{D 1}=0$ and $\beta_{D 2}=\pi / 2$ like in Fig. 1(c). The correlation between experimental results and the model confirms that the observed artefacts are generated by IP polarization. As tip length decreases, the model predicts a reduction of IP contribution to the OP signal due to the increase of longitudinal spring constant that reduces longitudinal bending transferred to the normal one. Using shorter tips significantly increases non-local electrostatic interaction [Fig. 3(a)]. In agreement with the model, it can be noticed in Fig. 4(b, c), that the overall OP amplitude is much larger for short tips. This intensity increase has been attributed to a larger contribution of non-local capacitive forces that appears as a deflection offset that has to be taken into account with local capacitive force to correctly estimate $\mathrm{d}_{33}$. The domains/domain walls contrast, estimated around $4 \pm 1 \mathrm{~nm}$ in Fig. $4(\mathrm{~b}, \mathrm{c})$ is in a fairly good agreement with our simulation that predicts a contrast of $3.6 \mathrm{~nm}$ for a d $_{33}=100 \mathrm{pm} / \mathrm{V} \cdot{ }^{\mathbf{2 4 , 2 5}}$ This contrast is used to determine $\mathrm{d}_{33}$, while the amplitude of domain walls gives access the offset deflection induced by local and non-local electrostatic forces. At the same time, too short tips $(<1 \mu \mathrm{m})$ can provokes polarization switching, since electric field may become significantly stronger and reach a threshold value. An optimum tip length in between 1 and $4 \mu \mathrm{m}$ is deduced to reduce IP contribution in the OP signal and avoid switching of domain polarization.

\section{SUMMARY}

In this work we have identified the limitations of cantilevers with long tips (15 $\mu$ m length) that prevent unambiguous determination of the orientation of FE domains and the OP piezoelectric coefficient by OP PFM in BFO thin films. The strong coupling of the cantilever OP oscillation with IP piezoresponse, through elastic forces, results in artefacts in the detected phase shift and amplitude signal for long tips. The contrast changes for different sample crystallographic orientations are related to the angle between the IP polarization vector and the cantilever beam. Up to $2 \mathrm{~nm}$ amplitude contrast can be induced by IP perturbations. 1D beam modelling of the cantilever oscillation amplitude in OP PFM was used to demonstrate that the IP contribution was the main artefact and an optimal tip length range $(1-4 \mu \mathrm{m})$ was estimated. A set of new cantilevers with different tip lengths were prepared by FIB and confirmed that IP contribution can be reduced and completely removed for tips shorter than $4 \mu \mathrm{m}$. Using tip shorter than $1 \mu \mathrm{m}$ led to domain polarization switching, defining a lower limit for the tip length. Optimizing the tip length to improve OP PFM sensitivity on BFO thin films can be applied to any other piezoelectric materials with similar piezoelectric properties where IP artefacts are present or suspected. 


\section{ACKNOWLEDGMENTS}

This work was conducted in collaboration with Materials and Condensed Matter Physics Group, University of Glasgow, UK and Research and Educational Centre "Nanotechnology" of Southern Federal University, Russia. We gratefully acknowledge Dr. Antoine Ruyter and Jean-Pierre Ouvrard for insightful participation in scientific discussions.

${ }^{1}$ S. Kalinin and A. Gruverman, (Scanning Probe Microscopy of Electrical and Electromechanical Phenomena at the Nanoscale. Springer Science \& Business Media, 2007).

${ }^{2}$ M. Alexe, A. Gruverman, (Nanoscale characterisation of ferroelectric materials: Scanning Probe Microscopy Approach. NanoScience and Technology. Springer, 2004).

${ }^{3}$ A. Gruverman, S. V. Kalinin, J. of Mat. Sci.41, 107-116 (2006).

${ }^{4}$ S. V. Kalinin, B. J. Rodriguez, S. Jesse, J. Shin, A. P. Baddorf, P. Gupta, H. Jain, D. B. Williams, and A. Gruverman, Microsc. Microanal. 12, 206-220 (2006).

${ }^{5}$ S. V. Kalinin, and D. A. Bonnell, Phys. Rev. B 65, 125408 (2002).

${ }^{6}$ S. Jesse, A. P. Baddorf, and S. V. Kalinin, Nanotechnology 17, 1615-1628 (2006).

${ }^{7}$ S. V. Kalinin, E. Karapetia, M. Kachanov, Phys. Rev. B 70, 184101 (2004).

${ }^{8}$ S. V. Kalinin, and D. A. Bonnell, J. Mater. Res. 17, 936-939 (2002).

${ }^{9}$ L. F. Henrichs, J. Bennett, and A. J. Bell, Rev. Sci. Instrum. 86, 083707 (2015).

${ }^{10}$ S. V Kalinin, A. N. Morozovska, L. Q. Chen, and B. J. Rodriguez, Rep. Prog. Phys. 73, 056502 (2010).

${ }^{11}$ R. Proksch, and S. Kalinin. Asylum Res. PFM, app note 10.

${ }^{12}$ A. Labuda and R. Proksch, Appl. Phys. Lett. 106, 253103 (2015).

${ }^{13}$ F. Johann, A. Morelli, and I. Vrejoiu, Phys. Status Solidi B 249, 2278-2286 (2012).

${ }^{14}$ F. Johann, A. Morelli, D. Biggemann, M. Arredondo, and I. Vrejoiu, Physical Review B 84, 094105 (2011).

${ }^{15}$ S. V. Kalinin, and A. Gruverman, (Scanning Probe Microscopy of Functional Materials. Springer Science \& Business Media, 2010).

${ }^{16} \mathrm{http}: / / w w w . n t m d t-t i p s . c o m$

${ }^{17}$ J. M. Neumeister, and W. A. Ducker, Rev. Sci. Instrum. 65, 2527 (1994).

${ }^{18}$ D.-A. Mendels, M. Lowe, A. Cuenat, M. G. Cain, E. Vallejo, D. Elli and F. Mendels, J. Micromech. Microeng. 16, 1720-1733 (2006).

${ }^{19}$ Y.Song, B. Bhushan. (Applied Scanning Probe Methods V. Scanning Probe Microscopy Techniques. NanoScience and Technology, Springer, 2006). 
${ }^{20}$ B. G. Konoplev, O. A. Ageev, V. A. Smirnov, A. S. Kolomiitsev, and N. I. Serbu, Rus. Microelectr. 41, 41-50 (2012).

${ }^{21}$ O. A. Ageev, A. S. Kolomiytsev, A. V. Bykov, V. A. Smirnov, and I. N. Kots, Microel. Reliab., 55, 2131-2134 (2015).

${ }^{22}$ N. Alyabyeva, A. Ouvrard, I. Lindfors-Vrejoiu, O. Ageev, and D. McGrouther, Appl. Phys. Lett. 111, 222901 (2017).

${ }^{23}$ S. M. Han, H. Benaroya, and T. Wei, J. of Sound and Vibration 225(5), 935-988 (1999).

${ }^{24}$ E. Finot, A. Passian, and T. Thundat, Sensors 8, 3497-3541 (2008).

${ }^{25}$ Y. F. Hou, W. L. Li, T. D. Zhang, W. Wang, W. P. Cao, X. L. Liu, and W. D. Fei, Phys. Chem. Chem. Phys. 17, 11593-11597 (2015).

${ }^{26}$ S. V. Kalinin, A. Gruverman, J. Shin, A. P. Baddorf, E. Karapetian, and M. Kachanov, Journal of Appl. Phys. 97, 074305 (2005). 